\title{
PHOTOLUMINESCENCE AND PHOTOACOUSTIC INVESTIGATIONS OF BERYLLIUM AND MAGNESIUM CONTAINING WIDE GAP II-VI MIXED CRYSTALS*
}

\author{
F. FIRSZT ${ }^{a}$, S. ŁęGOWSKI ${ }^{a}$, H. MĘCZYNSKA ${ }^{a}{ }^{\dagger}$, B. SEKULSKA ${ }^{a}$, \\ J. SzatKowski ${ }^{a}$, J. ZakrzeWsKI ${ }^{a}$ and W. PASZKowICZ ${ }^{b}$ \\ ${ }^{a}$ Institute of Physics, Grudziądzka 5/7, 87-100 Torun, Poland \\ ${ }^{b}$ Institute of Physics, Polish Academy of Sciences \\ Al. Lotników 32/46, 02-668 Warsaw, Poland
}

(Received September 15, 1998; revised version March 29, 1999)

$\mathrm{Zn}_{1-x} \mathrm{Be}_{x} \mathrm{Se}, \mathrm{Cd}_{1-x} \mathrm{Mg}_{x}$ Se and $\mathrm{Zn}_{1-x-y} \mathrm{Be}_{x} \mathrm{Mg}_{y}$ Se mixed crystals grown from the melt with different concentrations of $\mathrm{Be}$ and $\mathrm{Mg}$ have been characterized by photoluminescence and photoacoustic methods. An increase in band gap energy with increasing $\mathrm{Be}$ and $\mathrm{Mg}$ contents was observed. The photoacoustic spectroscopy was also employed for evaluation of thermal diffusivity of mixed $\mathrm{Zn}_{\mathrm{n}_{1-x}} \mathrm{Be}_{x}$ Se crystals with different beryllium contents.

PACS numbers: $46.30 . \mathrm{Pa}, 62.65 .+\mathrm{k}, 72.20 . \mathrm{My}, 78.20 . \mathrm{Hp}, 78.55 . \mathrm{Et}$

\section{Introduction}

The physical properties of wide gap II-VI ternary and quaternary mixed crystals have been recently extensively studied. These materials are of significant interest for their potential applications in technology of blue semiconductor lasers [1]. Structural and electrooptical properties of mixed crystals of $\mathrm{Mg}$ chalcogenides with wide gap II-VI compounds have been studied for a few years and $\mathrm{Zn}_{1-x} \mathrm{Mg}_{x} \mathrm{~S}_{1-y} \mathrm{Se}_{y}$ has been successfully used in construction of blue-green laser diode operating at room temperature (RT) with the lifetime of four hundred hours. Until now only a few papers concerning $\mathrm{Zn}_{1-x} \mathrm{Be}_{x} \mathrm{Se}, \mathrm{Cd}_{1-x} \mathrm{Mg}_{x} \mathrm{Se}$, and $\mathrm{Zn}_{1-x-y} \mathrm{Cd}_{x} \mathrm{Mg}_{y}$ Se bulk crystals have been published. $\mathrm{Zn}_{1-x} \mathrm{Be}_{x}$ Se has a much higher degree of covalent bonding and cohesive energy as compared to other more ionic wide gap II-VI binary compounds [2]. It has been recently shown that beryllium containing II-VI semiconductors such as $\mathrm{Zn}_{1-x} \mathrm{Be}_{x} \mathrm{Se}, \mathrm{Zn}_{1-x} \mathrm{Be}_{x} \mathrm{Te}$ and $\mathrm{Zn}_{1-x-y} \mathrm{Mg}_{x} \mathrm{Be}_{y}$ Se may offer a possibility of achieving a significant impact on the defect propagation and therefore the increase in the laser structure lifetime $[3,4]$.

*The results of this paper were initially presented at The Jablonski Centennial Conference on Luminescence and Photophysics, July 23-27, 1998, Toruń, Poland.

†e-mail: hmecz@phys.uni.torun.pl 
This is due to the observed noticeable lattice strengthening. The possibility of lattice matching of these Be-containing semiconductors to Si substrate is also very interesting. Some preliminary data concerning crystal structure and microhardness of $\mathrm{Zn}_{1-x} \mathrm{Be}_{x} \mathrm{Se}(0<x<0.17)$ bulk crystals have been already published [5,6]. Up to now however it is only a little known about radiative and nonradiative processes in beryllium containing wide gap II-VI mixed crystals.

This paper deals with investigations of photoluminescence (PL) and photoacoustic (PA) properties of $\mathrm{Zn}_{1-x} \mathrm{Be}_{x} \mathrm{Se}, \mathrm{Cd}_{1-x} \mathrm{Mg}_{x} \mathrm{Se}$ and $\mathrm{Zn}_{1-x-y} \mathrm{Be}_{x} \mathrm{Mg}_{y} \mathrm{Se}$ bulk crystals.

\section{Experiment}

Mixed crystals were grown from the melt by the high pressure Bridgman method under argon overpressure [5,6]. The crystals were cut into $1.0 \div 1.5 \mathrm{~mm}$ thick plates and mechanically polished. Some of them were annealed at $1230 \mathrm{~K}$ in zinc vapor for 48 hours. The chemical analysis was performed using an emission spectrometer with plasma excitation ICP2070 supplied by BAIRD. A standard $\mathrm{X}$-ray Bragg-Brentano powder diffractometer with $\mathrm{Ni}$-filtered $\mathrm{Cu} K_{\alpha}$ radiation was used for the phase analysis. Photoluminescence spectra were measured for temperatures ranging from $40 \mathrm{~K}$ up to $\mathrm{RT}$ in the energy limits from $1.6 \mathrm{eV}$ to $3.6 \mathrm{eV}$. Photoacoustic spectra were investigated using an open cell with continuous wave excitation. A $300 \mathrm{~W}$ xenon short arc lamp was used as the radiation source and its output made to pass through a prism monochromator. The PA signal was detected by a piezoelectric transducer (PZT) and analyzed with a lock-in amplifier.

\section{Results and discussion}

$$
\text { 3.1. } Z n_{1-i v} B e_{x} S e
$$

All investigated $\mathrm{Zn}_{1-x} \mathrm{Be}_{x}$ Se crystals for $0 \leq x \leq 0.44$ exhibit a sphalerite structure. The applied growth conditions and further annealing are observed to have influence on structural, electrical and luminescence properties of these crystals. The annealing of as-grown $\mathrm{Zn}_{1-x} \mathrm{Be}_{x}$ Se samples in zinc vapor at $1230 \mathrm{~K}$ improves their luminescence properties and for low beryllium content $(x<0.1)$ reduces the electric resistivity noticeably.

The PL spectra of $\mathrm{Zn}_{0.914} \mathrm{Be}_{0.086} \mathrm{Se}$ as-grown and annealed in zinc vapor are presented in Fig. 1. The spectrum at $T=40 \mathrm{~K}$ consists of a sharp line at $3.01 \mathrm{eV}$, a band at $2.88 \mathrm{eV}$ with a resolved LO phonon structure and two wide deep levels emission bands with maxima at $2.4 \mathrm{eV}$ and $2.1 \mathrm{eV}$. With increasing temperature the $2.88 \mathrm{eV}$ and $2.4 \mathrm{eV}$ PL bands are completely thermally quenched but the bands corresponding to the lowest $(2.1 \mathrm{eV})$ and to the highest $(3.01 \mathrm{eV})$ photon energies are observed up to room temperature. The nature of the latter cannot be exactly determined from the present limited data since the temperature dependence of the energy gap in $\mathrm{Zn}_{1-x} \mathrm{Be}_{x}$ Se mixed crystals is not exactly known as yet. However, taking into account the energetic position of this PL line at different temperatures $(40 \div 300 \mathrm{~K})$ and comparing these results with analogous data for ZnSe, we conclude that this emission can be due to recombination of free excitons associated with inelastic scattering with free carriers [7] or recombination of elec- 

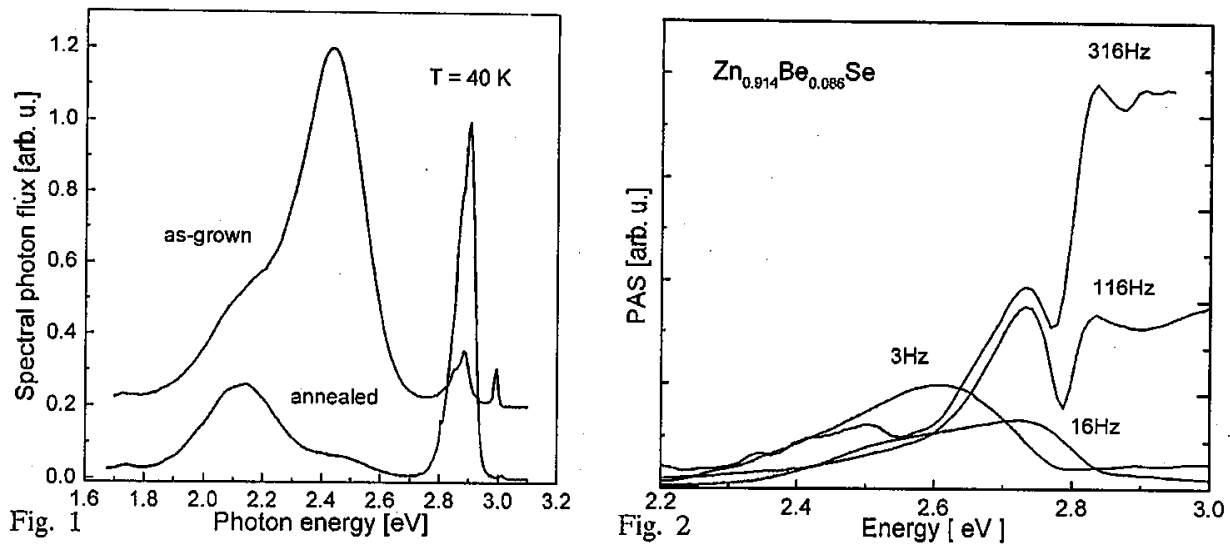

Fig. 1. Photoluminescence spectra at $T=4.0 \mathrm{~K}$ of $\mathrm{Zn}_{0.914} B \mathrm{e}_{0.086}$ Se crystals as-grown and annealed in zinc vapor.

Fig. 2. Photoacoustic spectra of $\mathrm{Zn}_{0.914} \mathrm{Be}_{0.086}$ Se at different frequencies of modulated radiation.

trons from shallow donors with free holes [8]. The band at $2.88 \mathrm{eV}$ (edge emission) is associated with free to bound transition or radiative recombination of shallow donor-acceptor pairs. The annealing of $\mathrm{Zn}_{1-x} \mathrm{Be}_{x} \mathrm{Se}$ in zinc vapor leads to an increase in the intensity ratio of near-band-edge emission to deep levels one. From photoluminescence measurements the exciton transition energy of $\mathrm{Zn}_{1-x} \mathrm{Be}_{x} \mathrm{Se}$ as a function of Be content was estimated.
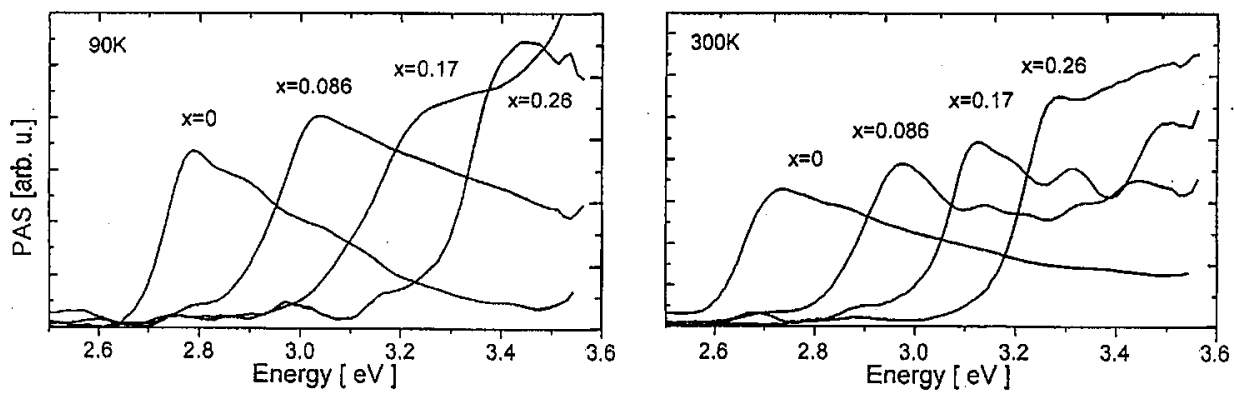

Fig. 3. Photoacoustic spectra of $\mathrm{Zn}_{1-x} \mathrm{Be}$ se annealed crystals with various $\mathrm{Be}$ contents at $90 \mathrm{~K}$ and $300 \mathrm{~K}$.

Figure 2 presents typical PA curves for as-grown $\mathrm{Zn}_{1-x} \mathrm{Be}_{x} \mathrm{Se}$ crystals at different frequencies of modulated radiation. The observed PA curves exhibit well-expressed changes of the slope at points from which the energy gap can be determined. It was observed that the position of these points is frequency dependent.

Figure 3 shows photoacoustic spectra at $90 \mathrm{~K}$ and $300 \mathrm{~K}$ for $\mathrm{Zn}_{1-x} \mathrm{Be} x \mathrm{Se}$ with different $B e$ concentrations annealed in zinc vapor. PA spectra for as-grown samples with the same Be content show a different behavior. It was observed that. 
the slope of PA curves does not change for different frequencies. In annealed samples a noticeable decrease in low frequencies of PA signal was observed for photon energies lower than the band gap energy. These results correlate with photoluminescence data indicating that the annealing of the $\mathrm{Zn}_{1-x} \mathrm{Be}_{x} \mathrm{Se}$ crystals in zinc causes a decrease in concentration of defect centers and thus an increase in crystal quality. In order to determine the optical band gap energy, the dependence of absorption coefficient on energy in the wavelength region above the fundamental absorption edge has to be considered. The absorption coefficients were determined from the experimental photoacoustic spectra. The Jackson-Amer model [9] of the piezoelectrically detected photoacoustic effect was applied to analyze the obtained spectra and the dependence of PA signal on absorption coefficient for different frequencies was calculated. The band gap energies estimated for investigated samples as a function of composition at $T=90 \mathrm{~K}$ and $T=300 \mathrm{~K}$ are presented in Fig. 4 . At the same figure exciton transition energies, obtained from PL spectra at $40 \mathrm{~K}$, are also shown for the same samples.
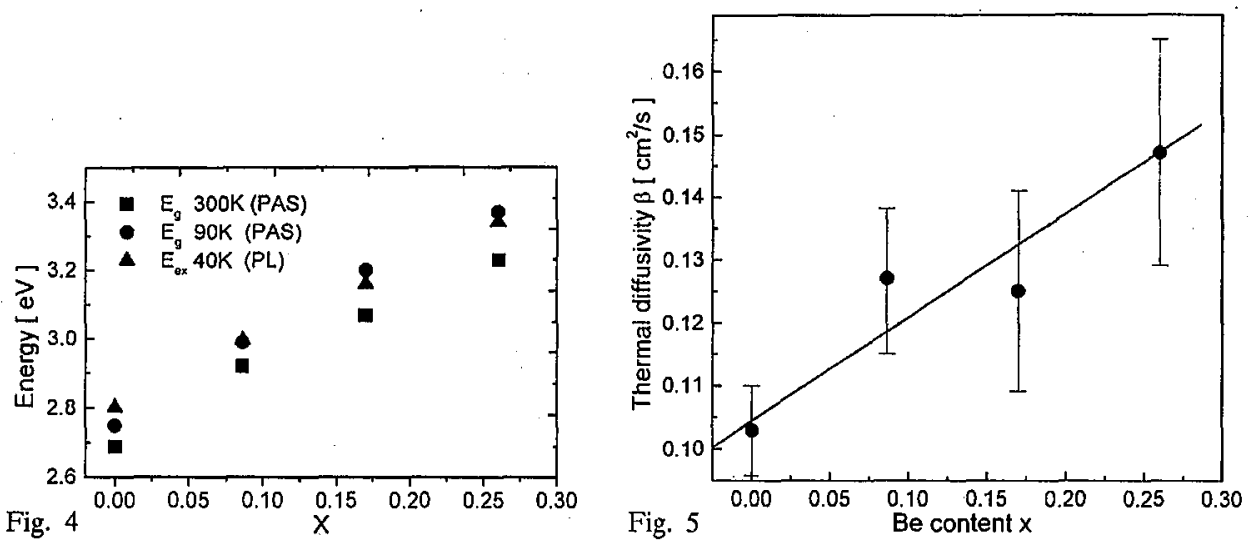

Fig. 4. Dependence of energy gap $\left(E_{\mathrm{g}}\right)$ and exciton transition energy $\left(E_{\text {ex }}\right)$ for $\mathrm{Zn}_{1-x} \mathrm{Be}_{x}$ Se crystals on composition.

Fig. 5. Dependence of thermal diffusivity of $\mathrm{Zn}_{1-x} \mathrm{Be}_{x} \mathrm{Se}$ crystals annealed in zinc vapor on composition.

From the analysis of the frequency dependence of the photoacoustic curves thermal diffusivity was derived: The thermal diffusivity is an especially important physical parameter, because it measures the time required to establish the thermal equilibrium in the sample. The thermal diffusivity is a unique parameter characteristic of each material like the energy gap. Following Blonskij et al. [10] we have examined the dependence of amplitude and phase of PA signal versus light modulation frequency. A single signal method with piezoelectric detection has been used to determine the thermal diffusivity. The samples were irradiated with modulated light in the strong absorption region. The thermal diffusivity was determined as a fitting parameter to the phase as well as to the amplitude of the signal. Figure 5 presents the dependence of the thermal diffusivity on composition of $\mathrm{Zn}_{1-x} \mathrm{Be}_{x} \mathrm{Se}$ crystals. 


\section{2. $C d_{1-x} M g_{x} S e$}

$\mathrm{Cd}_{1-x} \mathrm{Mg}_{x}$ Se mixed crystals form the hexagonal structure in the investigated range of compositions $(0<x<0.55)$. The typical PL spectrum at $40 \mathrm{~K}$ consists of near-band-edge (exciton), edge and deep levels emissions bands. The position of the highest photon energy line varies from $1.80 \mathrm{eV}$ for CdSe up to about $2.82 \mathrm{eV}$ for $\mathrm{Cd}_{1-x} \mathrm{Mg}_{x} \mathrm{Se}$ with $x \approx 0.55$. Figure 6 shows the photoluminescence spectra for pure CdSe and $\mathrm{Cd}_{1-x} \mathrm{Mg}_{x} \mathrm{Se}$ crystals at $40 \mathrm{~K}$ for several compositions: $x=0.0$, $0.15,0.30$ and 0.55 . The intensity ratio of exciton line to the edge emission increases with increasing $\mathrm{Mg}$ content. The latter was not observed in $\mathrm{Cd}_{1-x} \mathrm{Mg}_{x} \mathrm{Se}$ samples with $\mathrm{Mg}$ concentration higher than about $30 \%$. The edge emission band is thermally quenched at temperatures higher than about $150 \mathrm{~K}$ but the highest energy line is observed up to room temperature. Taking into account the temperature behavior of this line and analogous lines in $\mathrm{ZnSe}, \mathrm{CdSe}$ and $\mathrm{Zn}_{1-x} \mathrm{Mg}_{x} \mathrm{Se}$, one can conclude that it can be due to recombination of the free excitons after their interaction with free carriers or recombination of bound electrons with free holes.
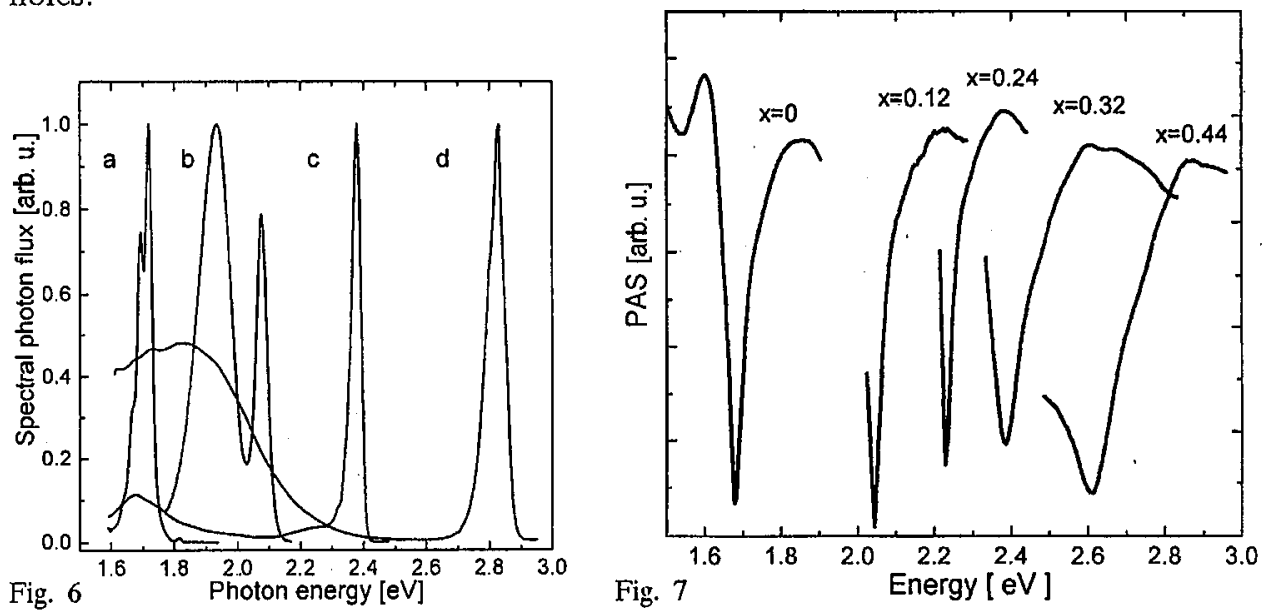

Fig. 6. Photoluminescence spectra of $\mathrm{Cd}_{1-x} \mathrm{Mg}_{x}$ Se crystals at $T=40 \mathrm{~K}$ for different Mg contents: (a) $x=0.0$, (b) $x=0.15$, (c) $x=0.30$, (d) $x=0.55$.

Fig. 7. Photoacoustic spectra of $\mathrm{Cd}_{1-x} \mathrm{Mg}_{x}$ Se at $T=300 \mathrm{~K}$ for different $\mathrm{Mg}$ contents.

In Fig. 7 photoacoustic spectra of pure CdSe and $\mathrm{Cd}_{1-x} \mathrm{Mg}_{x}$ Se for different $x$ values at $300 \mathrm{~K}$ are shown. In some $\mathrm{Cd}_{1-x} \mathrm{Mg}_{x}$ Se mixed crystals the photoacoustic signal at energies lower than $E_{\mathrm{g}}$ is larger than that for energies higher than the energy gap. This indicates that the PA signal does not increase monotonically with the optical absorption.

In order to obtain $E_{\mathrm{g}}$ values for $\mathrm{Cd}_{1-x} \mathrm{Mg}_{x}$ Se we consider the first derivative of the PA spectrum. The observed PA curves exhibit well-expressed changes of the slope at the points from which these energies are determined. It was found that in $\mathrm{Cd}_{1 .-x} \mathrm{Mg}_{x}$ Se the room temperature band gap energy ranges from $1.7 \mathrm{eV}$ to $2.7 \mathrm{eV}$ for $0<x<0.44$, respectively. 


$$
\text { 3.3. } Z n_{1-x-y} B e_{x} M g_{y} S e
$$

The quaternary $\mathrm{Zn}_{0.87} \mathrm{Be}_{0.03} \mathrm{Mg}_{0.10}$ Se crystal of sphalerite structure with a lattice constant ( $a=5.667 \AA$ ) nearly equal to that of ZnSe and energy gap $2.97 \mathrm{eV}$ was also grown. Photoluminescence spectra of such crystal at different temperatures are presented in Fig. 8. Assuming a linear dependence of the lattice constant

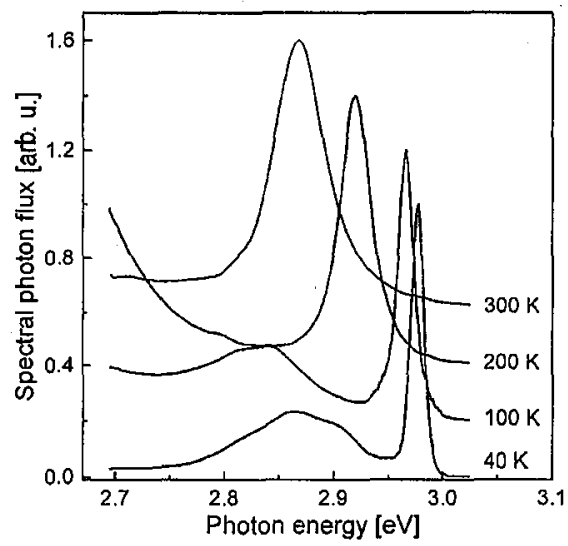

Fig. 8. Photoluminescence spectra of $\mathrm{Zn}_{0.87} \mathrm{Be}_{0.03} \mathrm{Mg}_{0.1}$ Se mixed crystal at different temperatures.

and band gap energy on composition for $\mathrm{Zn}_{1-x} \mathrm{Mg}_{x} \mathrm{Se}$ and $\mathrm{Zn}_{1-x} \mathrm{Be}_{x} \mathrm{Se}$ components, the calculated concentrations of the Be and $\mathrm{Mg}$ are $x=0.024$ and $y=0.080$. These values are almost equal to the real values $x=0.03$ and $y=0.10$ obtained from the chemical analysis.

This work was partly sponsored by the Committee for Scientific Research through the grant 2P03B12714.

\section{References}

[1] A.V. Nurmikko, R.L. Gunshor, Solid State Phys. 49, 205 (1996).

[2] C. Verie, Mater. Sci. Eng. B 43, 60 (1997).

[3] A. Waag, F. Fischer, H.J. Lugauer, Th. Litz, Th. Gerhard, J. Nurnberger, U. Lunz, U. Zahnder, W. Ossau, G. Landwehr, B. Ross, H. Richter, Mater. Sci. Eng. B 43, 65 (1997).

[4] A. Waag, F. Fischer, H.J. Lugauer, Th. Liz, J. Laubender, U. Lunz, U. Zehnder, W. Ossau, Th. Gerhard, M. Moller, G. Landwehr, J. Appl. Phys. 80, 792 (1996).

[5] F. Firszt, S. Łęgowski, H. Męczyńska, J. Szatkowski, W. Paszkowicz, K. Godwod, J. Cryst. Growth 184/185, 1335 (1998).

[6] W. Paszkowicz, K. Godwod, J. Domagała, F. Firszt, J. Szatkowski, H. Męczyńska, S. Eęgowski, M. Marczak, Solid State Commun. 107, 735 (1998).

[7] X.W. Fan, J. Woods, IEEE Trans. Electron, Dev., ED 28, 428 (1981).

[8] Y. Shirakawa, H. Kukimoto, J. Appl. Phys. 51, 2014 (1980).

[9] W. Jackson, N.M. Amer, J. Appl. Phys. 51, 3343 (1980).

[10] I.V. Blonskij, V.A. Tkhoryk, M.L. Shendeleva, J. Appl. Phys. 79, 3512 (1996). 